\title{
MEASUREMENT AND SIMULATION OF EQUATORIAL IONOSPHERIC PLASMA BUBBLES TO ASSESS THEIR IMPACT ON GNSS PERFORMANCE
}

\author{
Toshiaki Tsujii $^{1)}$. Takeshi Fujiwara ${ }^{2)} \cdot$ Tetsunari Kubota $^{3)}$. Chalermchon Satirapod ${ }^{4)}$. \\ Pornchai Supnithi $^{5)} \cdot$ Takuya Tsugawa $^{6)} \cdot$ Hungkyu Lee ${ }^{7)}$
}

\begin{abstract}
Ionospheric anomaly is one of the major error sources which deteriorate the GNSS performance. In the equatorial region, effects of the ionospheric plasma bubbles are of great interest because they are pretty common phenomena, especially in the period of the high solar activity. In order to evaluate the GNSS performance under circumstance of the bubbles, an ionospheric scintillation monitor has been developed and installed in Bangkok, Thailand. Furthermore, a model simulating the ionospheric delay and scintillation due to the bubbles has been developed. Based on these developments, the effects of the simulated plasma bubbles are analyzed and their agreement with the real observation is demonstrated. An availability degradation of the GPS ground based augmentation system (GBAS) caused by the bubbles is exampled in details. Finally, an integrated GPS/INS approach based on the Doppler frequency is proposed to remedy the deterioration.
\end{abstract}

Keywords : Ionospheric Plasma Bubble, Ionospheric Scintillation Monitor, GBAS, Availability, GPS/INS

\section{Introduction}

Although GNSS has been widely used for a great number of positioning/navigation/timing applications, its accuracy and reliability would not be sufficient under a harsh operational environment, such as the ionospheric anomaly. It is crucial for the safety-of-life applications, like aircraft aviation, to retain the reliability in any condition, which has led to developed GNSS augmentation systems. In Japan, the MSAS (MTSAT Satellite-based Augmentation System), compatible with the US WASS and European EGNOS systems, has been developed by the Japan Civil Aviation Bureau. The system has been operational for en-route and non-precision approach since September 2007. Furthermore, a prototype of GBAS for category-I precision approach has been developed by Electronic Navigation Research Institute (Yoshihara et al., 2010). However, even with such augmentation systems as depicted in Fig. 1, the GNSS availability might not be sufficient under the severe ionospheric scintillation and intentional/unintentional interference

It has been well known that the storm enhanced density (SED) associated with a severe magnetic storm yields a large special gradient of the ionospheric delay. However, the effect of the plasma bubble has to be considered in the countries

1) Corresponding Author, Associate Senior Researcher, Aviation Program Group, Japan Aerospace Exploration Agency, Japan (Email: tsujii.toshiake@jaxa.jp)

2) Researcher, Aviation Program Group, Japan Aerospace Exploration Agency, Japan

3) Researcher, Aviation Program Group, Japan Aerospace Exploration Agency, Japan

4) Professor, Department of Survey Engine ering, Chulalongkorn University, Thailand

5) Associate Professor, Faculty of Engineering, King Mongkut's Institute of Technology Ladkrabang, Bangkok, Thailand

6) Senior Researcher, Applied Electromagnetic Research Institute, National Institute of Information and Communications Technology, Japan

7) Associate Professor, Department of Civil Engineering, Changwon National University, Republic of Korea 
at relatively low magnetic latitude including Japan because it causes the scintillation as well as the spatial gradient (Saito et al., 2009). Therefore, GNSS performance evaluation under the ionospheric plasma bubbles is an important task in order to develop a new safety-of-life technology such as GBAS.

In this paper, a newly developed software-based scintillation monitor is firstly introduced. This is followed by description of the scintillation monitor installed in Bangkok, Thailand and the observations used to extract the characteristics of the plasma bubble. In addition, details of a mathematical model of the plasma bubbles developed and used for simulation of the GNSS operational environment are presented. As an example of GNSS performance assessment, an availability degradation of the GBAS induced by the plasma bubbles is analyzed. Finally, the GPS/INS integration approach is proposed as a candidate technology to retain the reliability under the adverse operational environment in that the Doppler-aiding by an inertial sensor is able to improves the receiver tracking performance and the so-called INScoasting maintains positioning continuity for a certain period.

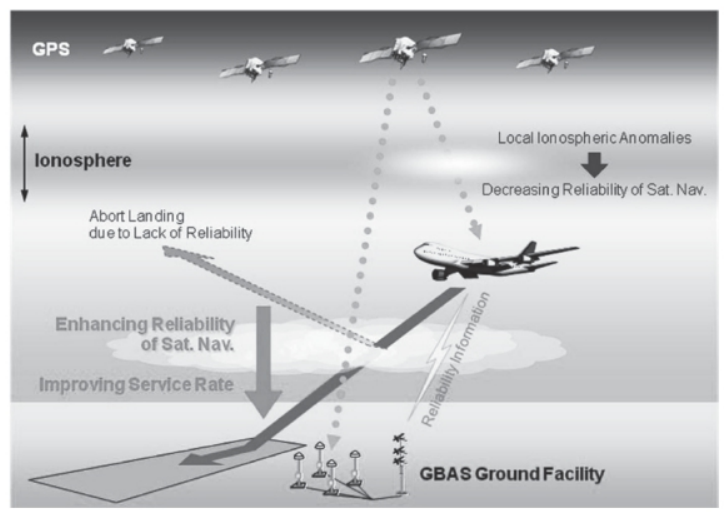

Fig. 1. Conceptual description of degraded availability of precision approach due to the ionospheric anomalies

\section{SOFTWARE-BASED IONOSPHERIC SCINTILLATION MONITOR}

An algorithm calculating the ionospheric scintillation indices has been implemented in a software GNSS receiver developed by iP-solutions Inc. (Petrovski and T. Tsujii, 2012). The receiver is basically consisted of two major components. One is a USB front end, which has functions to receive L1
GPS signals, down-convert and digitize them. The digitized signals are repacked and decimated if required and sent to PC through the USB with the sampling rate of 16 mega per second. The software component of the receiver includes baseband and navigation processing parts. The former comprises acquisition, tracking, and scintillation monitoring module. The comparison between the hardware receiver and software receiver is compared in Fig. 2.

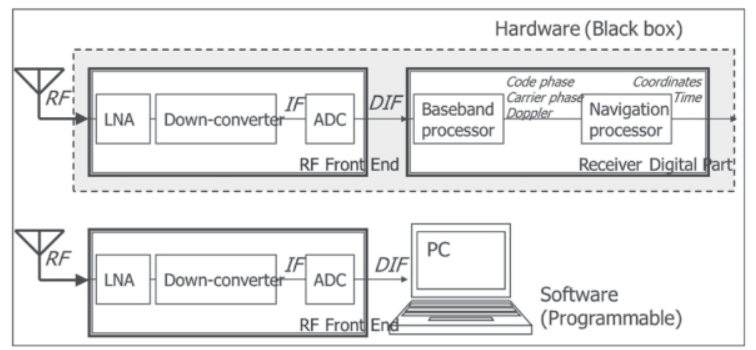

Fig. 2. Block diagrams of hardware and software receive

The main function of the scintillation monitor is to compute the scintillation indices such as $\mathrm{S}_{4}$ and carrier sigma $\left(\sigma_{\phi}\right)$ every minute. The index $\mathrm{S}_{4}$ gives the root mean square deviation of the power, normalized by the mean power.

$$
S_{4}=\sqrt{\frac{\left\langle P^{2}\right\rangle-\langle P\rangle^{2}}{\langle P\rangle^{2}}}
$$

where $<\bullet$ is an operator computing average of $\bullet$ values.

The signal power can be measured as a difference between narrow band $P_{N}$ and wide band power $P_{w}$ like (Van Dierendonck et al., 2004):

$$
P=P_{W}-P_{N}
$$

where the wide-band power is defined as:

$$
P_{W}=\sum_{j=1}^{M}\left(I_{j}^{2}+Q_{j}^{2}\right)
$$

and the narrow-band power as

$$
P_{N}=\left(\sum_{j=1}^{M} I_{j}\right)^{2}+\left(\sum_{j=1}^{M} Q_{j}\right)^{2}
$$

where $I_{j}$ and $Q_{j}$ are in-phase and quadra-phase samples $(1 \mathrm{kHz})$, and both powers are computed over $M(=20)$ samples. 
It is also necessary to remove a part of signal power coming from an ambient noise, which can be calculated by using signal to noise ratio (Van Dierendonck et al., 2004).

$$
S_{4 N}=\sqrt{\frac{100}{S / N_{0}}\left[1+\frac{500}{19 S / N_{0}}\right]}
$$

Finally, the $S_{4}$ index is computed as follows:

$$
S_{4}=\sqrt{\frac{\left\langle P^{2}\right\rangle-\langle P\rangle^{2}}{\langle P\rangle^{2}}-\frac{100}{S / N_{0}}\left[1+\frac{500}{19 S / N_{0}}\right]}
$$

The carrier phase measurement is de-trended by a highpass filter in order to get rid of low-frequency part including satellite motion. Subsequently, the standard deviation $\left(\sigma_{\phi}\right)$ is calculated over 60 seconds.

In order to present an example of plasma bubble measurement, Fig. 3 plots the Total Electron Content (TEC) and Rate of TEC Index (ROTI), which is defined as the standard deviation of ROT (rate of TEC), observed in Bangkok on September 22, 2011. It is of importance to note that the TEC bias is not calibrated in the results. The spatial scale of ROTI corresponds to a fairly large length (e.g., 6km), while that of $\mathrm{S}_{4}$ is about $400 \mathrm{~m}$ corresponding to the Fresnel size for GPS L1 frequency and an ionospheric height of $400 \mathrm{~km}$ (Basu et al., 1999). In the dotted circle, lots of TEC depletions and due to plasma bubbles and ROTI values are observed in the corresponding period.

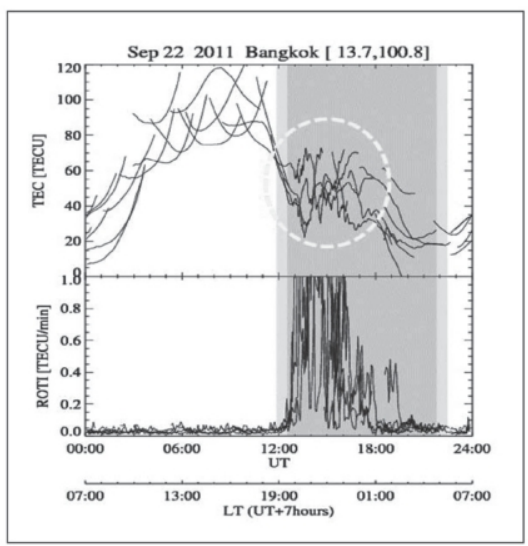

Fig. 3. TEC/ROTI measured in Bangkok (http:wdc.nict.go.jp/ION02/THAI-GPS/KMI/ROTI)

The TEC value of PRN 29 is picked up from Fig. 3 and presented at the top graph in Fig. 4. Three successive TEC depletions caused by three bubbles can be seen in the graph. The $\mathrm{S}_{4}$ values computed by the software-based Ionospheric scintillation monitor (ISM) are shown in the middle graph, indicating the strong correlation with the TEC variation Since the elevation angle of PRN 29 is not low as shown in the bottom graph, the large $\mathrm{S}_{4}$ value would not be caused by the GNSS multipath. Fig. 5 depicts the ionospheric delay of PRN 29 on September 20 to compensate the orbit difference for two days (e.g., two times of $3 \mathrm{~min} 56 \mathrm{sec}$ ). Since the ROTI values on the September 20 were low, there seems to be no bubble on the day. However, it clear that TEC depletion and large $\mathrm{S}_{4}$ values in Fig. 4 are caused by the plasma bubbles

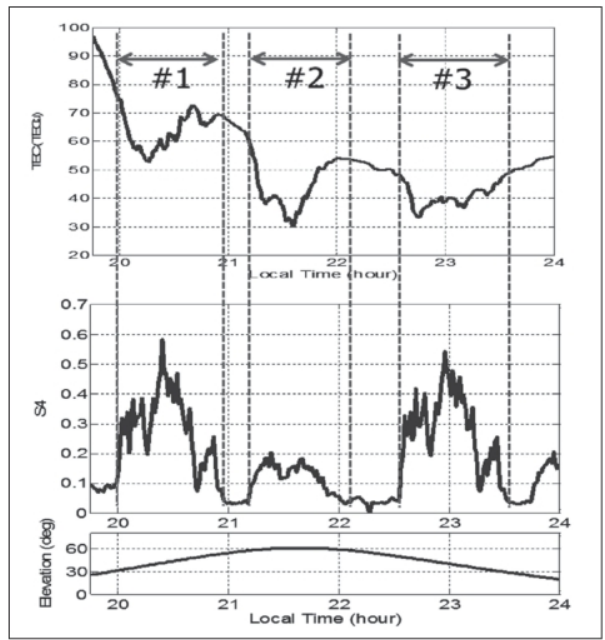

Fig. 4. TEC and $\mathrm{S}_{4}$ for PRN 29 (Three bubbles are observed)

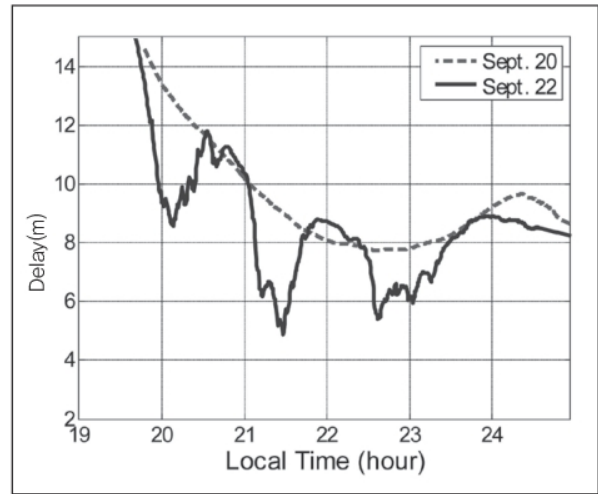

Fig. 5. Ionospheric delays for PRN 29 on September 20 and $\mathbf{2 2}$ (Biases of delays are not calibrated) 


\section{SIMULATION OF PLASMA BUBBLE}

In order to access the impact of the plasma bubble into GNSS performance, an ionospheric environmental simulation tool has been developed in this study. An analytical model of the electron density profile, the so-called NeQuick model, is used as the background density model. Furthermore, the three dimensional plasma bubble model developed by ENRI is used (Saito et al., 2009). Fig. 6 shows the cross section of the bubble model.

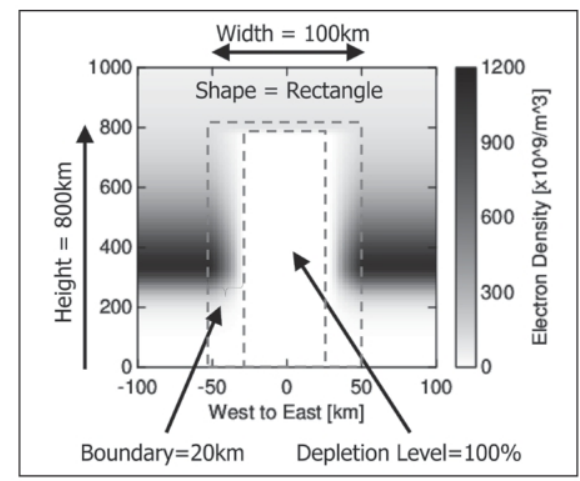

Fig. 6. Plasma bubble model

Four plasma bubbles are embedded to simulate the ionospheric environment described in the previous section. In Fig. 7, the bubbles are shown by thin lines in the dotted circle. They are moving eastward towards Bangkok with a typical speed $(100 \mathrm{~m} / \mathrm{s})$. The resultant ionospheric delay observed in Bangkok is compared with the real data in Fig. 8 , manifesting the agreement of the tendencies of gradients and depletion depths. Hence, it is verified from there results that the developed simulation tool is adequate to evaluate the ionospheric effects on GNSS signals.

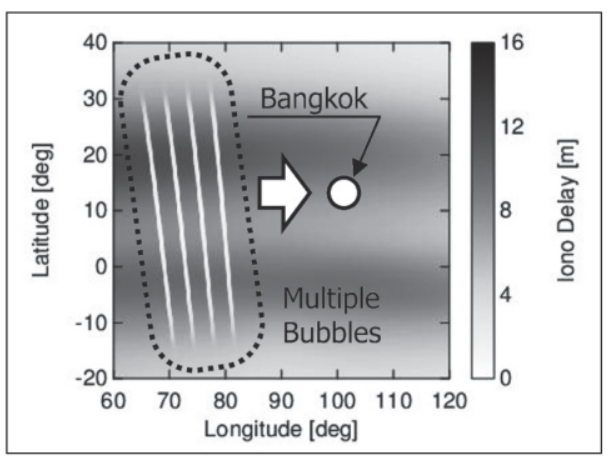

Fig. 7. Simulated ionospheric delay with plasma bubbles

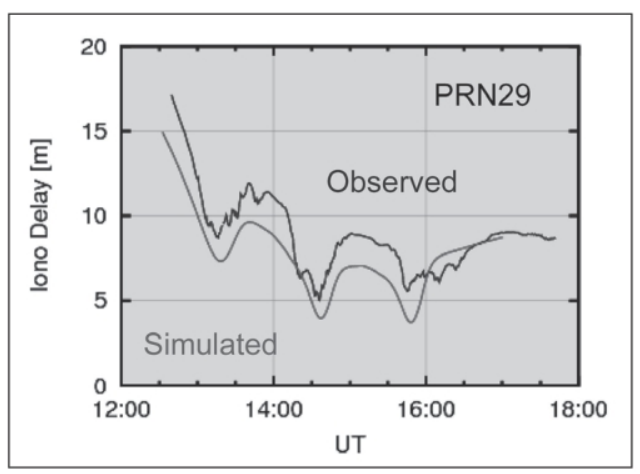

Fig. 8. Simulated ionospheric delay of PRN 29 compared with the observed value

\section{EFFECT OF PLASMA BUBBLES ON GBAS AVAILABILITY}

An availability degradation of GBAS caused by the plasma bubbles is evaluated in this section. The vertical protection level (VPL) of GAST-D (GBAS Approach Service Type D, providing a guidance for CAT-III operations) for a day in March in Bangkok without the plasma bubble is computed as shown in Fig. 9. The effective ionization level parameter (e.g., $\mathrm{Az}$, of NeQuick model) is set at 200 with a consideration of high solar activity, and twenty four GPS full-constellation is assumed according to $\mathrm{DoD}$ (2008). In addition, high accuracy of GPS signals is assumed for both ground (GAD-C) and airborne (AAD-B) subsystems (RTCA, 2004; RTCA, 2008). Additional parameters requisite for VPL calculation include: (a) maxSvert (a maximum vertical dilution extracted from

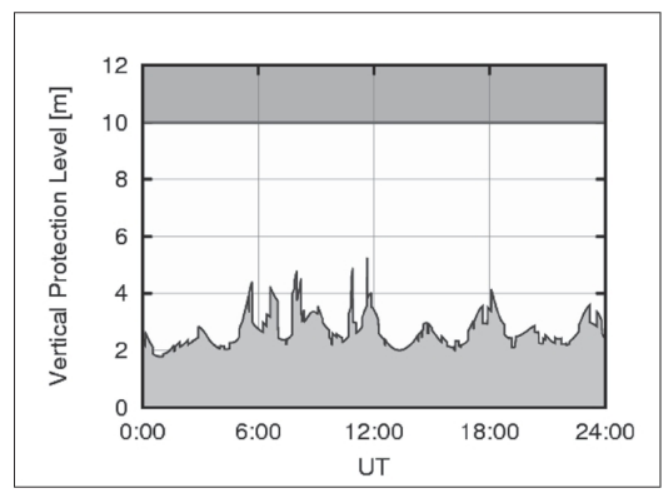

Fig. 9. Vertical protection level of GBAS GAST-D for a day in March at Bangkok(Without plasma bubble) 
the projection matrix of the least squares estimation) for geometry screening is four; (b) distance between aircraft and GBAS reference point is $5 \mathrm{~km}$; (c) approach speed is 70 $\mathrm{m} / \mathrm{s}$; (d) sigma of vertical ionospheric gradient $\left(\sigma_{v i g}\right)$ is $4 \mathrm{~mm} /$ $\mathrm{km}$. If VPL is below the vertical alert limit (VAL), which is defined as 10 meters in this simulation, the GAST-D service is available. Therefore, the availability of GAST-D is $100 \%$ if the plasma bubble does not occur.

As presented in Fig. 8, a large gradient of the ionospheric delay appears when the plasma bubbles intercept the GPS signal propagation. Since the ionospheric gradient affects the differential GPS positioning performance, the amplitude of gradient has to be monitored by the so-called codecarrier divergence (CCD) monitor. If the monitored value exceeds a threshold, the corresponding satellite should be excluded from the navigation computation. Furthermore, the scintillation usually occurs in the bubble, and the GPS signal is fluctuated when it propagates into the bubble. When the scintillation is strong, the GPS signal lock would be lost, so that positioning error and VPL increase. In order to access the effect of the lock loss for the GBAS availability, JAXA has developed a GPS lock loss model as depicted in Fig. 10. A second-order phase lock loop (PLL) is implemented, and the Monte Carlo simulations with several $\mathrm{S}_{4}$ values were carried out to obtain the probability of the lock loss. The Cornell scintillation model is used of which the correction time is set at 0.2 seconds (Humphreys et al., 2009). Since the $\mathrm{S}_{4}$ of the generated signal data differs from the specified $\mathrm{S}_{4}$, the input $\mathrm{S}_{4}$ values are adjusted. Therefore, the $\mathrm{S}_{4}$ of the generated

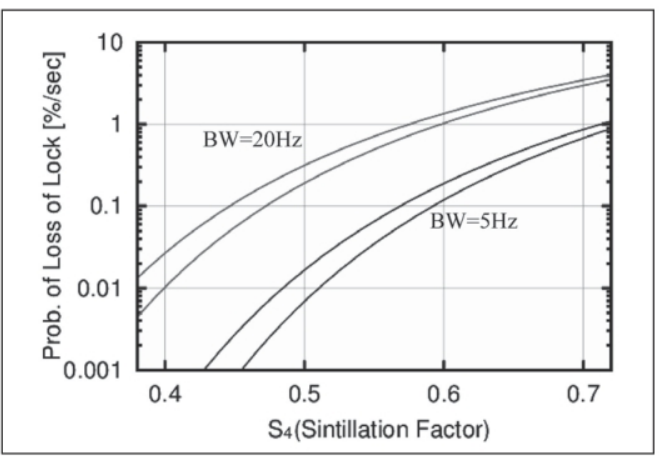

Fig. 10. GPS lock loss model as a function of $\mathrm{S}_{4}$ with PLL bandwidth (BW) of $\mathbf{2 0}$ and $\mathbf{5 H z}$ (Upper: low elevation satellites; Lower: high elevation satellite) data becomes the intended values. The probabilities of the loss of lock with PLL bandwidth of $20 \mathrm{~Hz}$ and $5 \mathrm{~Hz}$ are shown in the figure. The upper lines for the both bandwidths represent a low elevation satellite $\left(\mathrm{C} / \mathrm{N}_{0}=37.5 \mathrm{~dB}-\mathrm{Hz}\right)$ while the lower lines indicate a high elevation satellite $\left(\mathrm{C} / \mathrm{N}_{0}=52.0\right.$ $\mathrm{dB}-\mathrm{Hz}$ ). Since an airborne GPS receiver has to track aircraft dynamics, a wider bandwidth is usually used (15-25 Hz). If $\mathrm{S}_{4}$ is 0.6 , rate of the lock loss for the receiver with bandwidth of $20 \mathrm{~Hz}$ is about once a 100 seconds, and the signal would not be used for the navigation computation.

The four plasma bubbles are assumed as described in the previous section. Since some satellites are excluded by the CCD monitor or the signal lock loss due to the scintillation, the VPL increased in accordance with the passages of plasma bubbles and exceeded the VAL in some periods, degrading the availability of GAST-D to $98.0 \%$ as shown in Fig. 11 .

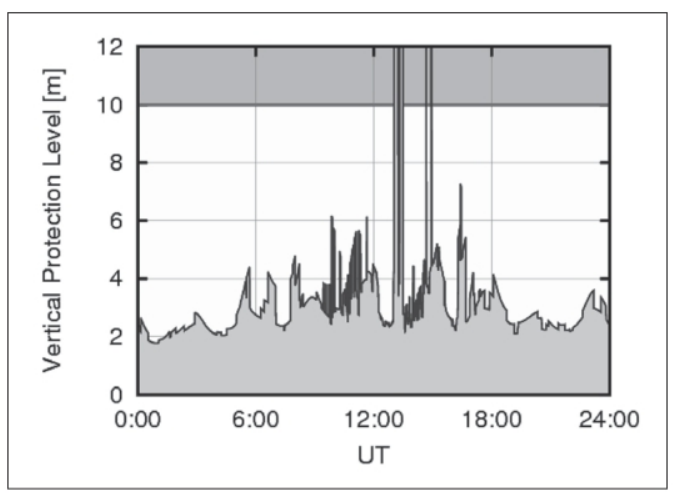

Fig. 11. Vertical protection level of GBAS GAST-D for a day in March at Bangkok (with four plasma bubbles)

\section{IMPROVEMENT OF GBAS AVAILABILITY BY GPS/INS}

In order to improve the satellite availability under the harsh ionospheric environment, JAXA has been developing a technology of GPS and inertial integrated system (GPS/ INS). Detainment of the carrier tracking is crucial for the aircraft precision approach using GBAS because the carrierphase observations are used to smooth the pseudorange. If cycle slips occur in several channels, the corresponding smoothing procedures have to be restarted, resulting in a missed approach. The tracking performance can be improved 
by aiding an inertial sensor since the Doppler frequency shift caused by aircraft dynamics can be compensated by its measurements. Fig. 12 shows the block diagram of Doppleraided tracking loop. As presented in the figure, $e$ in the equation is the unit vector from aircraft to satellite.

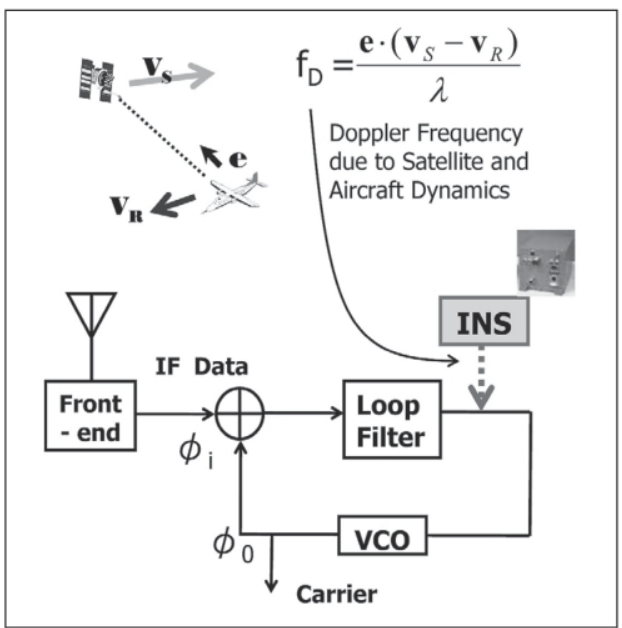

Fig. 12. Block diagram of Doppler-aided tracking loop

Previous researches using realflight data have demonstrated that the Doppler-aided airborne receiver enabled to track GPS signal with a narrow PLL bandwidth of $3 \mathrm{~Hz}$. Therefore, 5 Hz PLL bandwidth would be adequate for the aided airborne receiver under the scintillation. The GPS/INS has also the

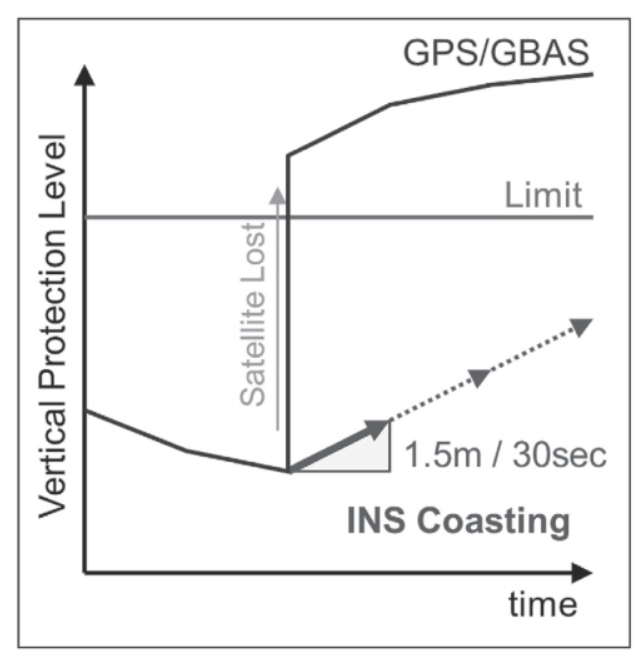

Fig. 13. Conceptual sketch of INS-Coasting advantage which makes it possible to continuously perform the navigation computation during a certain period of GPS signal outage. This function is called 'INS-coasting' (see, Fig. 13) and the maximum positioning error after the GPS outage is 1.5 meters $/ 30 \mathrm{sec}$ for a navigation grade inertial sensor (RTCA, 2011). When several satellites are excluded either by the CCD monitor or by the loss of the lock and the number of satellites is insufficient, the vertical protection level abruptly exceeds VAL so that the GBAS availability is lost. However, if the INS-coasting is applied, the precision approach can be continued during the period because the increased rate of VPL is less than 1.5 meters $/ 30$ seconds.

The vertical protection level is recalculated by applying the inertial measurements for the GPS/SBAS as presented in Fig. 14. Considering the advantage of the GPS/INS integration, it is obvious from the results that the availability of GAST-D is improved up to $99.1 \%$.

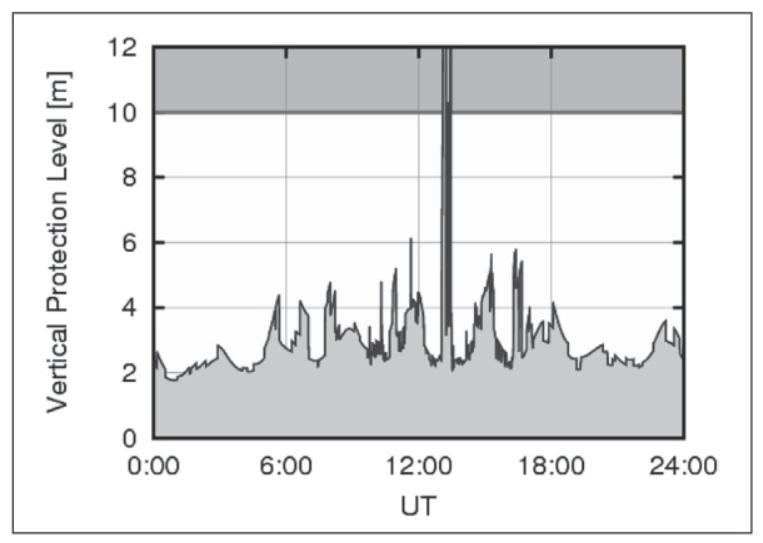

Fig. 14. Vertical protection level of INS-aided GBAS GAST-D for a day in March at Bangkok (with four plasma bubbles assumed in Fig. 7)

\section{CONCLUDING REMARKS}

This paper deals with impact of the equatorial ionospheric plasma bubble into the GNSS positioning/navigation performance. To this end, the newly developed softwarebased ionospheric scintillation monitor has been introduced with a brief description of the ionospheric scintillation monitor installed in Bangkok, Thailand. Based on the real world data observed at the monitor, an ionospheric 
environmental model to simulate plasma bubbles has been developed in this research. An availability degradation of GPS/GBAS under the bubbles was detailed with an emphasis of their impact into satellite-tracking performance. In addition, effects of including the inertial measurements into the receiver-tracking loop have demonstrated improvement of the GNAS availability. Finally, it is under a consideration to include the observations collection/evaluation in the next solar maximum period (2013-2014) to refine the simulation tools, which more realistically model the plasma bubbles

\section{References}

Basu, S., Groves, K.M., Quinn, J.M. and P. Doherty (1999), A comparison of TEC fluctuations and scintillation as Ascension Island, Journal of Atmospheric and SolarTerrestrial Physics, Vol. 61, pp. 1219-1226.

Di Giovanni, G. and Radicella, S.M. (1990), An Analytical Model of the Electron Density Profile in the Ionosphere, Advances in Space Research, Vol. 10, No. 11, pp. 27-30.

Humphreys, T.H., Psiaki, M.L., Hinks, J.C., O'Hanlon, B. and Kintner, P.M. (2009), Simulating IonosphereInduced Scintillation for Testing GPS Receiver Phase Tracking Loops, IEEE Journal of Selected Topics in Signal Processing, Vol. 3, No. 4, pp. 707-715.

Tsujii, T., Fujiwara, T., Suganuma, Y., Tomita, H. and Petrovski, I. (2011), Development of INS-Aided GPS Tracking Loop and Flight Test Evaluation, SICE Journal of Control, Measurement, and System Integration, Vol. 4, No. 1, pp. 015-021.

Saito S, Yoshihara, T. and Fujii, N. (2009), Study of Effects of the Plasma Bubble on GBAS by a ThreeDimensional Ionospheric Delay Model, Proceedings of ION-GNSS2009, Savannah, GA, September 22-25, pp. 1141-1148
Van Dierendonck, A. J., and Arbesser-Rastburg, B. (2004), Measuring Ionospheric Scintillation in the equatorial Region Over Africa, Including Measurement From SBAS Geostationary Satellite Signals, Proceedings of ION GNSS-2004, Long Beach, CA, September 21-24, pp. 316-324.

Petrovski, I. and Tsujii, T. (2012), Digital Satellite Navigation and Geophysics -A Practical Guide with GNSS Signal Simulator andReceiver Laboratory, Cambridge University Press, Cambridge, UK, p. 338

Yoshihara, T., et al., Kudo, T., Fukushima, S., Saitoh, S., Saito, S., Fujita, S., Fujii, N. and Hoshinoo, K. (2010), Development of GBAS (Ground-Based Augmentation System) prototype for safety design and evaluation, IEICE Technical Report SSS2010-3 (2010-05), The Institute of Electronics, Information and Communication Engineers(in Japanese)

DoD (2008), GPS Standard Positioning Service Performance Standard, $4^{\text {th }}$ Edition, US Department of Defense, Washington, DC, p. 160

ICAO NSP/WGW (2010), GBAS CAT II/III Development Baseline SARPs, Working Paper 59, p. 212

RTCA (2004), Minimum Aviation Performance for GPS Local Area Augmentation System (LAAS), DO-245A.

RTCA (2008), Minimum Operational Performance Standards for GPS Local Area Augmentation System Airborne Equipment, DO-253C.

RTCA (2011), Information Paper (Version 7), SC-159 WG$2 \mathrm{C}$.

(Received October 23, 2012; Revised October 24, 2012; Accepted December 17, 2012) 